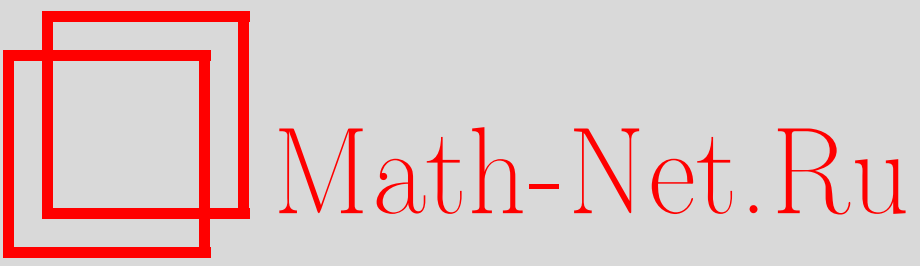

С. В. Шадрин, Числа Гурвица обобщенных многочленов и циклы двухточечных ветвлений, УМН, 2003, том 58, выпуск 1, 197-198

DOI: https://doi.org/10.4213/rm606

Использование Общероссийского математического портала Math-Net.Ru подразумевает, что вы прочитали и согласны с пользовательским соглашением

http://www.mathnet.ru/rus/agreement

Параметры загрузки:

IP: 52.87 .193 .239

26 апреля 2023 г., 09:59:14 


\title{
ЧИСЛА ГУРВИЦА ОБОБЩЕННЫХ МНОГОЧЛЕНОВ И ЦИКЛЫ ДВУХТОЧЕЧНЫХ ВЕТВЛЕНИЙ
}

\author{
С.В. ШАДРИн
}

1. Обобщенными многочленами мы назьваем мероморфные функции на компактных римановых поверхностях произвольного рода такие, что прообраз точки $\infty \in \mathbb{C P}^{1}$ состоит ровно из одной точки.

Определим числа Гурвица обобщенных многочленов с одним не простым критическим значением. Для этого нужно зафиксировать род $g$, степень $n$ и разбиение $\left(a_{1}, \ldots, a_{l}\right)$ числа $n$. Затем мы отметим на $\mathbb{C P}^{1}$ попарно различные точки $z_{1}, \ldots, z_{2 g+l+1}, z_{1}=\infty$.

Числом Гурвица $h\left(g, n ; a_{1}, \ldots, a_{l}\right)$ назьвается число обобщенных многочленов $f: C \rightarrow \mathbb{C P}^{1}$ степени $n$, определенных на кривых рода $g$, таких, что $z_{3}, \ldots, z_{2 g+l+1}$ - простые критические значения $f$ и $f^{-1}\left(z_{2}\right)=a_{1} x_{1}+\cdots+a_{l} x_{l}\left(x_{1}, \ldots, x_{l}\right.$ - попарно различные точки $\left.C\right)$.

При этом мы считаем функции $f_{1}: C_{1} \rightarrow \mathbb{C P}^{1}$ и $f_{2}: C_{2} \rightarrow \mathbb{C P}^{1}$ совпадающими, если найдется биголоморфное отображение $\phi: C_{1} \rightarrow C_{2}$ такое, что $f_{1}=f_{2} \phi$. Кроме того, многочлен $f: C \rightarrow$ $\mathbb{C P}^{1}$ учитывается с весом $c$, где $1 / c=|\operatorname{aut}(f)|$.

2. Напомним, что через $\overline{\mathscr{M}}_{g, k}$ обозначается компактификация пространства модулей кривых рода $g$ с $k$ упорядоченными отмеченньми точками. Через $\psi_{i}$ обозначается первый класс Черна расслоения $L_{i} \rightarrow \overline{\mathscr{M}}_{g, k}$ со слоем, равным кокасательной прямой в $i$-й отмеченной точке.

Рассмотрим набор целых чисел $c_{1}, \ldots, c_{k}$ такой, что $\sum_{i=1}^{k} c_{i}=0$. Обозначим через $V\left(g ; c_{1}, \ldots, c_{k}\right) \subset \overline{\mathscr{M}}_{g, k}$ замыкание подмногообразия $V^{0}\left(g ; c_{1}, \ldots, c_{k}\right) \subset \mathscr{M}_{g, k}$, состоящего из кривых $\left(C, x_{1}, \ldots, x_{k}\right)$ таких, что $\sum_{i=1}^{k} c_{i} x_{i}$ - дивизор мероморфной функции. Класы гомологий, определенные подмногообразиями $V\left(g ; c_{1}, \ldots, c_{k}\right)$, называются циклами двухточечных ветвлений (two-pointed ramification cycles).

3. Числа Гурвица можно выразить через пересечения с циклами двухточечных ветвлений.

Tеорема 1. Если $2 g+l \geqslant 2, m o$

$$
h\left(g, n ; a_{1}, \ldots, a_{l}\right)=\frac{n^{2 g+l-2}(2 g+l-1) !}{\operatorname{aut}\left(a_{1}, \ldots, a_{l}\right)} \int_{V\left(g ;-n, a_{1}, \ldots, a_{l}\right)} \psi_{1}^{2 g+l-2} .
$$

Например, если $g=0$, то $V\left(g ;-n, a_{1}, \ldots, a_{l}\right)=\overline{\mathscr{M}}_{0, l+1}$ и $\int_{\bar{M}_{0,1+l}} \psi_{1}^{l-2}=1$. Таким образом, мы получаем формулу $h\left(0, n ; a_{1}, \ldots, a_{l}\right)=n^{l-2}(l-1) ! / \operatorname{aut}\left(a_{1}, \ldots, a_{l}\right)$, совпадающую с соответствующим частным случаем формулы из [1].

Изложим (нестрого) схему доказательства теоремы 1 . Рассмотрим $\left(\mathbb{C P}^{1}, z_{1}, \ldots, z_{2 g+l+1}\right)$ как точку пространства модулей $\overline{\mathscr{M}}_{0,2 g+l+1}$. Функции, участвующие в определении чисел Гурвица, образуют пространство $H$. Определено отображение $l l: H \rightarrow \overline{\mathscr{M}}_{0,2 g+l+1}$, сопоставляющее функции набор ее критических значений. Отображение $l l$ - это разветвленное накрытие, и число Гурвица - это его степень.

Заметим, что $\int_{\bar{M}_{0,2 g+l+1}} \psi_{1}^{2 g+l-2}=1$. Следовательно, $h\left(g, n ; a_{1}, \ldots, a_{l}\right)=\int_{H} l l^{*} \psi_{1}$. Существует отображение $s t: H \rightarrow \overline{\mathscr{M}}_{g, N}$, сопоставляющее функции кривую в ее прообразе с отмеченными всеми прообразами всех критических значений $(N=1+l+(n-1)(2 g+l-1))$. Согласно лемме 1.17 в [2] $l l^{*} \psi_{1}=n \cdot s t^{*} \psi_{1}$.

Таким образом, мы получили выражение для $h\left(g, n ; a_{1}, \ldots, a_{l}\right)$ через $\int_{s t_{*}[H]} \psi_{1}^{2 g+l-2}$ с некоторым комбинаторным коэффициентом. Далее несложные геометрические соображения позволяют свести это выражение к формуле (1). Подробно это доказательство написано в [3].

Работа выполнена при частичной поддержке Российского фонда фундаментальных исследований (грант № 01-01-00660), NWO (грант № 047.008.005) и INTAS (грант № 00-0259). 
4. Можно также выразить числа пересечений через числа Гурвица. Обозначим через $h(g, n)$ число $h(g, n ; 1, \ldots, 1)=h(g, n ; 2,1, \ldots, 1)$.

Теорема 2. Для каждого $L \geqslant 0$ верно следующее:

$$
\int_{\overline{\mathcal{M}}_{g, 3}} \psi_{1}^{3 g}=\sum_{i=0}^{g}(-1)^{i}\left(\begin{array}{c}
g \\
i
\end{array}\right) \frac{(g+L+1-i) ! \cdot h(g, g+L+1-i)}{g !(3 g+L-i) !(g+L+1-i)^{3 g+L-1-i}} .
$$

Например, зная, что $\int_{\bar{M}_{g, 3}} \psi_{1}^{3 g}=1 /\left(24^{g} g !\right)$, и вычислив первые $g$ чисел Гурвица $h(g, 1), \ldots$, $h(g, g)$, нетрудно, пользясь этой формулой, вычислить любое другое число $h(g, k)$.

Проверим эту формулу при $g=1$. Напомним, что согласно частному случаю формулы из [4] $h(1, k)=(k+1) k^{k}(k-1) / 24$. Подставив это выражение в формулу $(2)$, мы получим, что $\int_{\overline{\mathscr{M}}_{1,3}} \psi_{1}^{3}=1 / 24$.

Заметим, что и в общем случае теорему 2 можно вьвести из формулы из [4] чисто комбинаторными рассуждениями, см. [3]. Однако наша цель сейчас - показать, как эту формулу можно увидеть из чисто геометрических соображений, ползуясь теоремой 1.

5. Обозначим через $V(g, n ; k, m) \subset \overline{\mathscr{M}}_{g, 1+k+m}$ замькание подмногообразия $V^{0}(g, n ; k, m)$ $\subset \mathscr{M}_{g, 1+k+m}$, состоящего из кривых $\left(C, x_{1}, \ldots, x_{1+k+m}\right)$ таких, что найдется обобщенный многочлен степени $n$, имеющий полюс в точке $x_{1}$ и нули в точках $x_{2}, \ldots, x_{1+k}$.

Заметим, что если $0 \leqslant n-k \leqslant g$, то размерность $V(g, n ; k, m)$ равна $2 g+n+m-2$. Кроме того, заметим, что $V(g, g+L+1 ; L+1, m)=\overline{\mathscr{M}}_{g, 2+L+m}$ для любого $L \geqslant 0$.

Лемма 1. При $0 \leqslant n-k \leqslant g$ интеграл $\int_{V(g, n ; k, m)} \psi_{1}^{2 g+n+m-2}$ не зависит от $m$.

Эта лемма является чем-то вроде частного случая уравнения струны, но только для интегралов по $V(g, n ; k, m)$. Доказьвается она так же, как и уравнение струны.

Лемма 2. При $0 \leqslant n-k \leqslant g$ мы имеем

$\int_{V(g, n ; k, m+1)} \psi_{1}^{2 g+n+m-1}=\int_{V(g, n ; k+1, m)} \psi_{1}^{2 g+n+m-2}-\int_{V(g, n-1 ; k, m+1)} \psi_{1}^{2 g+n+m-2}$.

Эта лемма вьводится из леммы 1.17 в [4] рассуждениями, сходными с доказательством теоремы 1.

Теперь заметим, что $\int_{\bar{M}_{g, 3}} \psi_{1}^{3 g}=\int_{V(g, g+L+1 ; L+1, g)} \psi_{1}^{4 g+L-1}$ (это следует из уравнения струны и из леммы 1$)$. Далее, многократным применением леммы 2 мы получаем, что

$$
\int_{V(g, g+L+1 ; L+1, g)} \psi_{1}^{4 g+L-1}=\sum_{i=0}^{g}(-1)^{i}\left(\begin{array}{c}
g \\
i
\end{array}\right) \int_{V(g, g+L+1-i ; g+L+1-i, i)} \psi_{1}^{3 g+L-1} .
$$

Тогда, пользуясь леммой 1 и теоремой 1 , мы получаем формулу (2). Подробно это доказательство приведено в [3].

6. Автор выражает благодарность С. К. Ландо, С. М. Натанзону и М.З. Шапиро за полезные обсуждения.

\section{СПИСОК ЛИТЕРАТУРЫ}

[1] С. К. Ландо, Д. Звонкин // Функц. анализ и его прил. 1999. Т. 33. № 3. С. 21-34. [2] E.-N. Ionel // Invent. Math. 2002. V. 148. № 3. P. 627-658; math.AG/99-08060. [3] S. V . Shadrin // math.AG/0209282. [4] T. Ekedahl, S. Lando, M. Shapiro, A. Vainshtein // Invent. Math. 2001. V. 146. № 2. P. 297-327.

Московский государственный университет им. М. В. Ломоносова, Независимьй московский университет 